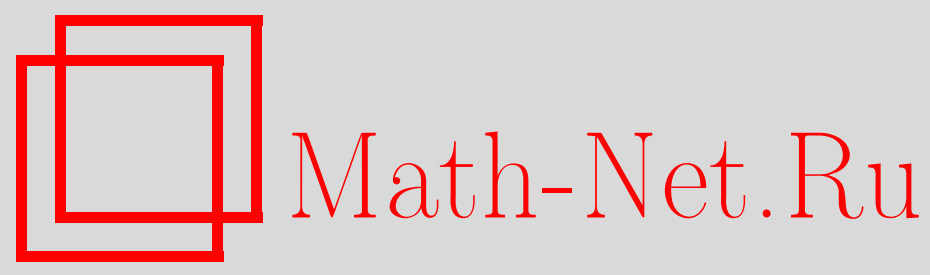

А. А. Болибрух, O tau-функции уравнения изомонодромных деформаций Шлезингера, Матем. заметки, 2003, том 74, выпуск 2, 184-191

DOI: https://doi.org/10.4213/mzm254

Использование Общероссийского математического портала Math-Net.Ru подразумевает, что вы прочитали и согласны с пользовательским соглашением http://www.mathnet.ru/rus/agreement

Параметры загрузки:

IP : 35.173 .219 .12

26 апреля 2023 г., 12:50:46

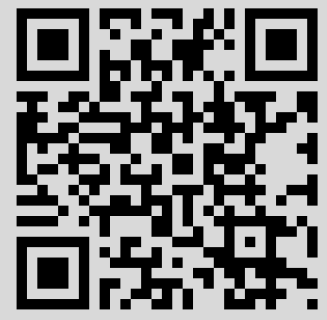


УДК 514

\section{О tau-ФУНКЦИИ УРАВНЕНИЯ ИЗОМОНОДРОМНЫХ ДЕФОРМАЦИЙ ШЛЕЗИНГЕРА}

\section{А. А. Болибрух}

В данной работе tau-функция для уравнения изомонодромных деформаций Шлезингера представляется в виде результата последовательных элементарных калибровочных преобразований, что, в частности, позволяет получить простое доказательство теоремы Мивы о tau-функции.

Библиография: 6 названий.

Введение. Рассмотрим уравнение Шлезингера изомонодромных деформаций

$$
d B_{i}(a)=-\sum_{j=1, j \neq i}^{n} \frac{\left[B_{i}(a), B_{j}(a)\right]}{a_{i}-a_{j}} d\left(a_{i}-a_{j}\right)
$$

с начальньми условиями

$$
\left.B_{i}(a)\right|_{a^{0}}=B_{i}^{0}
$$

Это уравнение является условием совместности для следующего изомонодромного семейства:

$$
d y=\left(\sum_{i=1}^{n} \frac{B_{i}(a)}{z-a_{i}} d\left(z-a_{i}\right)\right) y
$$

фуксовых систем дифференциальных уравнений на сфере Римана, где параметр $a=$ $\left(a_{1}, \ldots, a_{n}\right)$ принадлежит универсальному накрытию $Z$ пространства

$$
\mathbb{C}^{n} \backslash \bigcup_{i \neq j}\left\{a_{i}-a_{j}=0\right\} .
$$

Напомним, что изомонодромное семейство Шлезингера выделяется среди других изомонодромных семейств следующими свойствами [1]):

1) $\sum_{i=1}^{n} B_{i}(a)=$ const (и эта постоянная матрица равна нулю, если бесконечность является точкой голоморфности);

2) матрищы связи между некоторой фиксированной фундаментальной матрицей $Y(z, a)$ семейства $(3)$ и локальными левелевскими базисами в особых точках не зависят от $a$.

Хорошо известно (см. [2]), что для каждых начальных данных (2) уравнение (1) имеет единственное решение $B_{1}(a), \ldots, B_{n}(a)$ в некотором шаре $D\left(a^{0}\right)$. В [2] доказано следующее

Работа поддержана Российским фондом фундаментальных исследований, грант № 02-01-01286, а также грантом "Научные школы", № НШ-457.2003.1. 
УТВЕРЖДЕНИЕ 1. Решения $B_{1}(a), \ldots, B_{n}(a)$ могут быть продолэсены на все универсальное накрытие $Z$ пространства $\mathbb{C}^{n} \backslash \bigcup_{i=1}^{n}\left\{a_{i}-a_{j}=0\right\}$ как мероморфные функиии переменных $а=\left(a_{1}, \ldots, a_{n}\right)$.

Особые точки продолженных функций $B_{1}(a), \ldots, B_{n}(a)$ назьваются подвижсными особыми точками уравнения Шлезингера (потому что их положение зависит от начальньх условий (2)). Следовательно, последнее утверждение может быть сформулировано следующим образом.

УТВЕРЖДЕНИЕ 1'. Решения уравнения изомонодромных деформаций Шлезингера не имеют других подвижных особых точек кроме полюсов.

В таком случае говорят, что уравнение Шлезингера удовлетворяет свойству Пенлеве.

Обозначим через $\Theta$ множество подвижных особых точек уравнения $(1),(2)$. Это аналитическое подмножество множества $Z$ коразмерности один (либо это пустое множество, что также возможно).

Систему (3) (более точно, дифференциальную форму ее коэффициентов) можно рассматривать как плоскую связность $\nabla$ в голоморфном векторном расслоении $F$ над $T=$ $\overline{\mathbb{C}} \times Z$ (в фиксированной мероморфной тривиализации этого расслоения) ([2], см. также [3]). Отметим здесь, что ограничение этого расслоения на $\overline{\mathbb{C}} \times\left\{a^{0}\right\}$ является голоморфно тривиальньм и, стало быть, его степень равна нулю при $a=a_{0}$. Поскольку при изомонодромной деформации собственные значения матриц вычетов связности не меняются, расслоение $F$, ограниченное на $\overline{\mathbb{C}} \times\{a\}$, имеет нулевую степень для любой точки $a$ (напомним, что степень такого расслоения равна сумме всех собственных значений матриц вычетов связности по всем особым точкам).

Из сказанного вьше получаем следующее

УТВЕРЖДЕНИЕ 2 [2]. Множество $\Theta$ состоит из точек $a^{*} \in \Theta$, в которых расслоение $\left.F\right|_{\left(\overline{\mathbb{C}} \times\left\{a^{*}\right\}\right)}$ является голоморфно нетривиальным.

Согласно теореме Мивы [4] множество $\Theta$ является множеством полюсов формы

$$
\frac{1}{2} \sum_{i, j} \frac{\operatorname{tr}\left(B_{i}(a) B_{j}(a)\right) d\left(a_{i}-a_{j}\right)}{a_{i}-a_{j}} .
$$

Ниже мы приводим простой способ вычисления этого исключительного множества $\Theta$.

1. Вспомогательная система. Рассмотрим точку $a^{*} \in \Theta$ и мероморфную тривиализацию расслоения $\left.F\right|_{\left(\overline{\mathbb{C}} \times\left\{a^{*}\right\}\right)}$, голоморфную вне бесконечности и такую, что вьполняются следующие условия:

1) соответствующая система

$$
\frac{d y}{d z}=\left(\sum_{i=1}^{n} \frac{{ }^{*} B_{i}}{z-a_{i}^{*}}\right) y
$$

(которая определяется введенной связностью в выбранном базисе сечений) имеет фуксову особенность в бесконечности;

2 ) сумма $\sum_{i=1}^{n}{ }^{*} B_{i}$ равна $K=\operatorname{diag}\left(k_{1}, \ldots, k_{p}\right)$, где $k_{1} \leqslant \cdots \leqslant k_{p}$, матрица $-K$ задает тип расщепления расслоения $\left.F\right|_{\left(\overline{\mathbb{C}} \times\left\{a^{*}\right\}\right)}($ типом расщепления расслоения на римановой сфере назьвается набор степеней линейньх расслоений, прямой суммой которых является наше расслоение, см. [5]) и $\operatorname{tr} K=0$ (так как степень рассматриваемого расслоения равна нулю); 
3) одна из фундаментальных матриц системы в бесконечности имеет вид

$$
Y^{*}(z)=U(z) z^{K}, \quad U(z)=I+U_{1} \frac{1}{z}+U_{2} \frac{1}{z^{2}}+\cdots,
$$

причем дополнительно

$$
U(z) z^{K}=z^{K} V(z)
$$

для некоторой голоморфно обратимой в бесконечности матричной функции $V(z)$.

Существование подобного рода тривиализации доказано в [6].

Пусть $c_{1}<\cdots<c_{t}-$ различные числа из совокупности $\left\{k_{1}, \ldots, k_{p}\right\}$. Тогда $K=$ $\operatorname{diag}\left(c_{1} I_{1}, \ldots, c_{t} I_{t}\right)$, где размер соответствующей единичной матрицы $I_{j}$ совпадает с кратностью числа $c_{j}$. Для произвольной матрицы $A$ рассмотрим разбиение этой матрицы на блоки $A^{l m}$ в соответствии с введенной блочной структурой матрицы $K$. Тогда из (6) получаем, что для любых $m>l$ вьполнено

$$
U_{i}^{l m}=0, \quad \text { если } i<c_{m}-c_{l} .
$$

Включим построенную фуксову систему в изомонодромное семейство Шлезингера

$$
d y=\left(\sum_{i=1}^{n} \frac{{ }^{*} B_{i}(a)}{z-a_{i}} d\left(z-a_{i}\right)\right) y
$$

и обозначим через $Y(z, a)$ изомонодромную фундаментальную матрищу этого семейства вида

$$
Y(z, a)=U(z, a) z^{K}, \quad U(z, a)=I+U_{1}(a) \frac{1}{z}+U_{2}(a) \frac{1}{z^{2}}+\cdots
$$

в бесконечности и такую, что $Y(z, a)$ совпадает с $Y^{*}(z)$ из (5) для $a=a^{*}$.

Так как

$$
d_{a} Y(z, a) Y^{-1}(z, a)=-\sum_{i=1}^{n} \frac{{ }^{*} B_{i}(a) d a_{i}}{z-a_{i}}
$$

то из (8) следует, что

$$
\begin{aligned}
& d_{a} U_{1}(a) \frac{1}{z}+d_{a} U_{2}(a) \frac{1}{z^{2}}+\cdots=\left(-\left(\sum_{i=1}^{n}{ }^{*} B_{i}(a) d a_{i}\right) \frac{1}{z}\right. \\
& \left.\quad-\left(\sum_{i=1}^{n}{ }^{*} B_{i}(a) a_{i} d a_{i}\right) \frac{1}{z^{2}}+\cdots\right)\left(I+U_{1}(a) \frac{1}{z}+U_{2}(a) \frac{1}{z^{2}}+\cdots\right) .
\end{aligned}
$$

И мы получаем

$$
\begin{aligned}
d_{a} U_{1}= & -\left(\sum_{i=1}^{n}{ }^{*} B_{i}(a) d a_{i}\right), \ldots \\
d_{a} U_{j}= & -\left(\sum_{i=1}^{n}{ }^{*} B_{i}(a) a_{i}^{j-1} d a_{i}\right) \\
& -\left(\sum_{i=1}^{n}{ }^{*} B_{i}(a) a_{i}^{j-2} d a_{i}\right) U_{1}-\cdots-\left(\sum_{i=1}^{n}{ }^{*} B_{i}(a) d a_{i}\right) U_{j-1} .
\end{aligned}
$$

Для дальнейшего нам понадобится следующее утверждение. 
Лемма 1. Среди әлементов блоков ${ }^{*} B_{i}^{l m}(a), i=1, \ldots, n$, с индексами $l, m$ та-

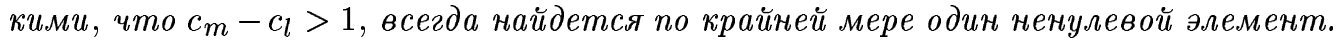

ДокАЗАТЕЛЬСТво. Предположим, что все элементы рассматриваемьх блоков тождественно равны нулю. Тогда для каждого фиксированного $a$ калибровочное преобразование $\tilde{Y}(z, a)=z^{-K} Y(z, a)$ преобразует нашу систему (7) в систему, не имеющую особенности в бесконечности, потому что матрица коэффициентов этой преобразованной системы

$$
\frac{d \widetilde{Y}}{d z}(\tilde{Y})^{-1}=\frac{-K}{z}+z^{-K}\left(\sum_{i=1}^{n} \frac{{ }^{*} B_{i}(a)}{z-a_{i}}\right) z^{K}
$$

имеет там нуль более, чем первого порядка. На самом деле, из построения следует только, что преобразованная система будет фуксовой в бесконечности с нильпотентной матрицей коэффициентов. Но так как матрица монодромии в бесконечности равна единичной, то матрица коэффициентов действительно равна нулю.

Но голоморфность преобразованной системы в бесконечности означает вьполнение для всех $а$ следующего соотношения, сходного с (6):

$$
U(z, a) z^{K}=z^{K} V(z, a)
$$

для некоторой голоморфно обратимой матричной функции $V(z, a)$. Но в свою очередь, это соотношение означает, что тип расшепления расслоения $F$ определяется диагональными элементами матрицы $-K$ для всех $a$, что противоречит голоморфной тривиальности расслоения $F$ вне множества $\Theta$.

СледСТВИЕ 1. Среди әлементов матрицы $U_{1}(a)$ из блоков $U_{1}^{l m}$ с индексами $l, m$ такими, что $c_{m}-c_{l}>1$, найдется по крайней мере один не равный тожсдественно нулю.

ДокАЗАТЕЛЬСТво следует немедленно из первого соотношения в (9).

2. Особая часть локальной tau-функции. Пусть ненулевой элемент из следствия 1 имеет индексы $l, m$.

Поскольку

$$
d_{z} Y(z, a) Y^{-1}(z, a)=\sum_{i=1}^{n} \frac{{ }^{*} B_{i}(a) d z}{z-a_{i}}
$$

получаем из (8)

$$
\begin{aligned}
& \left(-U_{1}(a) \frac{1}{z^{2}}+o\left(z^{-2}\right) z^{K}+\left(I+U_{1}(a) \frac{1}{z}+o\left(z^{-1}\right) \frac{K}{z} z^{K}\right.\right. \\
& =\left(\frac{K}{z}+\left(\sum_{i=1}^{n}{ }^{*} B_{i}(a) a_{i}\right) \frac{1}{z^{2}}+o\left(z^{-2}\right)\right)\left(I+U_{1}(a) \frac{1}{z}+o\left(z^{-1}\right)\right) z^{K} .
\end{aligned}
$$

Следовательно,

$$
-U_{1}+\left[U_{1}, K\right]=\sum_{i=1}^{n}{ }^{*} B_{i}(a) a_{i}
$$

Поэтому

$$
u_{1}^{l m}(a)=c b_{1}(a), \quad \text { где } \quad c=\frac{1}{k_{m}-k_{l}-1}, \quad b_{1}(a)=\sum_{i=1}^{n}{ }^{*} b_{i}^{l m}(a) a_{i} .
$$


Рассмотрим матрицу $\Gamma_{1}^{\prime}(z, a)=I+\mathscr{E}_{m l}$, у которой все элементы матрицы $\mathscr{E}_{m l}$ равны нулю за исключением элемента с индексами $m, l$, который равен $-z /\left(c b_{1}(a)\right)$. Учитьвая вид матрицы $U_{1}(a)$, получаем, что матрица $U^{\prime}(z, a)=\Gamma_{1}^{\prime} U(z, a)$ выглядит следующим образом:

$$
U^{\prime}(z, a)=\left(U_{0}^{\prime}(a)+U_{1}^{\prime}(a) \frac{1}{z}+o\left(z^{-1}\right)\right) z^{L},
$$

где $L$ - диагональная матрица с двумя ненулевыми элементами: 1 на месте $(l, l)$ и -1 на месте $(m, m)$.

Рассмотрим матрицу $\Gamma_{1}=\left(U_{0}^{\prime}(a)\right)^{-1} \Gamma_{1}^{\prime}(z, a)$. Калибровочное преобразование $Y_{1}(z, a)=\Gamma_{1}(z, a) Y(z, a)$ преобразует нашу систему в фуксову систему вида (3) с матрицей коэффищиентов $B_{i}^{1}(a)$ и такую, что матрица $Y_{1}(z, a)$ имеет в бесконечности разложение вида (8), где все вовлеченные матрицы снабжены теперь верхним индексом 1 и $K^{1}=K+L$. Заметим, что это разложение имеет место лиш вне некоторого аналитического подмножества $\Theta_{1}$ коразмерности один, содержащего точку $a^{*}$ (это множество является множеством нулей функции $\left.b_{1}(a)\right)$.

Обозначим через $|K|$ число $|K|=\sum_{i=1}^{p}\left(k_{i}\right)^{2}$. Тогда согласно приведенному построению

$$
\left|K^{1}\right| \leqslant|K|-2
$$

Действительно,

$$
\left|K^{1}\right|-|K|=\left(k_{l}+1\right)^{2}+\left(k_{m}-1\right)^{2}-k_{l}^{2}-k_{m}^{2}=2\left(1+k_{l}-k_{m}\right) \leqslant-2 .
$$

Если $K^{1} \neq 0$, то из того, что $\operatorname{tr} K^{1}=0$, следует существование по крайней мере двух элементов $k_{i}^{1}, k_{j}^{1}$ матрицы $K^{1}$, удовлетворяющих условию $k_{i}^{1}-k_{j}^{1} \geqslant 2, i>j$, и таким образом, процедуру, описанную вьше, можно применить еще раз. Через конечное число $s$ шагов получим фуксово семейство вида (3), голоморфное в бесконечности. Согласно теореме единственности для $(1),(2)$ эта итоговая система будет калибровочно эквивалентна исходной системе (3) с помощью постоянного калибровочного преобразования. И мы получаем

УТВЕРЖДЕНИЕ 3. Особое множсество $\Theta$ в окрестности точки а* совпадает $c$ множеством нулей функиии

$$
\tau^{*}(a)=\prod_{j=1}^{s} b_{j}(a)
$$

где $b_{j}(a)$ появляется на $j$-ом шаге процедуры, описанной выше, таким же образом, как и функиия $b_{1}(a)$.

Отметим здесь, что частньй случай вычисления tau-функции, похожий на приведенный выше, представлен в [2].

Для иллюстрации приведенного метода рассмотрим следующий пример. Фуксова система

$$
\frac{d y}{d z}=\left(\frac{1}{z}\left(\begin{array}{cc}
0 & -3 \\
0 & 0
\end{array}\right)+\frac{1}{z-1}\left(\begin{array}{cc}
0 & 3 \\
-3 & 0
\end{array}\right)+\frac{1}{z+1}\left(\begin{array}{cc}
-1 & 1 \\
-1 & 1
\end{array}\right)+\frac{1}{z-2}\left(\begin{array}{cc}
0 & -1 \\
4 & 0
\end{array}\right)\right) y
$$


имеет особые точки $a_{1}^{*}=0, a_{2}^{*}=1, a_{3}^{*}=-1, a_{4}^{*}=2$ и $\infty$. Калибровочное преобразование $y^{\prime}=z^{K} y$, где $K=\operatorname{diag}(1,-1)$, преобразует эту систему в систему

$$
\frac{d y^{\prime}}{d z}=\left(\frac{1}{z}\left(\begin{array}{cc}
1 & 0 \\
3 & -1
\end{array}\right)+\frac{1}{z-1}\left(\begin{array}{cc}
0 & 3 \\
-3 & 0
\end{array}\right)+\frac{1}{z+1}\left(\begin{array}{cc}
-1 & 1 \\
-1 & 1
\end{array}\right)+\frac{1}{z-2}\left(\begin{array}{cc}
0 & -4 \\
1 & 0
\end{array}\right)\right) y^{\prime} .
$$

Последняя система голоморфна в бесконечности. Поэтому систему (13) можно рассматривать как вспомогательную систему (4) для некоторого изомонодромного семейства, которое будет построено позже.

Действуя так же, как и в п. 1, включим эту систему в изомонодромное семейство Шлезингера (7). Введем следующие обозначения:

$$
t_{1}=a_{1}, \quad t_{2}=a_{2}-1, \quad t_{3}=a_{3}+1, \quad t_{4}=a_{4}-2 .
$$

Используя соотношение (1), нетрудно вычислить разложения в ряд Тейлора в точке $a^{*}$ для каждого ${ }^{*} B_{i}(a)$. В частности, для элементов ${ }^{*} b_{i}^{12}(a)$ этих матриц получаем

$$
\begin{array}{ll}
{ }^{*} b_{1}^{12}=-3+6 t_{1}-6 t_{3}+o(|t|), & { }^{*} b_{2}^{12}=3-3 t_{2}+3 t_{3}+o(|t|), \\
{ }^{*} b_{3}^{12}=1-6 t_{1}+3 t_{2}+\frac{11}{3} t_{3}-\frac{2}{3} t_{4}+o(|t|), & { }^{*} b_{4}^{12}=-1-\frac{2}{3} t_{3}+\frac{2}{3} t_{4}+o(|t|) .
\end{array}
$$

Поэтому,

$$
\begin{aligned}
b_{1}(t) & ={ }^{*} b_{1}^{12}(t) t_{1}+{ }^{*} b_{2}^{12}(t)\left(1+t_{2}\right)+{ }^{*} b_{3}^{12}(t)\left(-1+t_{3}\right)+{ }^{*} b_{4}^{12}(t)\left(2+t_{4}\right) \\
& =3 t_{1}-3 t_{2}-t_{3}+t_{4}+o(|t|) .
\end{aligned}
$$

В переменных $a_{1}, \ldots, a_{4}$ имеем

$$
b_{1}(a)=3 a_{1}-3 a_{2}-a_{3}+a_{4}+o\left(\left|a-a^{*}\right|\right) .
$$

Другими словами, мы вычислили касательную гиперплоскость в точке $a^{*}=$ $(0,1,-1,2)$ к особому множеству $\Theta$ для изомонодромного семейства (3), которое было получено из семейства (7) с помощью калибровочного преобразования $\Gamma_{1}^{\prime}(z, a)$, описанного вьше.

Действуя таким же образом, нетрудно вычислить $m$-струю функции $b_{1}(a)$ для любого $m$.

3. Элементарное доказательство теоремы Мивы. Как выразить функцию $\tau^{*}$ и множество $\Theta$ в терминах матриц коэффициентов $B_{i}(a)$ исходного семейства (3) (другими словами, как вьвести теорему Мивы из соотношения (12))? Рассмотрим вновь первый шаг процедуры, описанной вьше.

Имеем

$$
\begin{aligned}
\left(\sum_{i=1}^{n} \frac{B_{i}^{1}(a)}{z-a_{i}}\right)= & \frac{\partial \Gamma_{1}}{\partial z} \Gamma_{1}^{-1}+\Gamma_{1}\left(\sum_{i=1}^{n} \frac{{ }^{*} B_{i}(a)}{z-a_{i}}\right) \Gamma_{1}^{-1} \\
\left(\sum_{i=1}^{n} \frac{B_{i}^{1}(a)}{z-a_{i}}\right)^{2}= & \frac{\partial \Gamma_{1}}{\partial z}\left(\sum_{i=1}^{n} \frac{{ }^{*} B_{i}(a)}{z-a_{i}}\right)\left(\Gamma_{1}\right)^{-1}+\Gamma_{1}\left(\sum_{i=1}^{n} \frac{{ }^{*} B_{i}(a)}{z-a_{i}}\right)\left(\Gamma_{1}\right)^{-1} \frac{\partial \Gamma_{1}}{\partial z}\left(\Gamma_{1}\right)^{-1} \\
& +\Gamma_{1}\left(\sum_{i=1}^{n} \frac{{ }^{*} B_{i}(a)}{z-a_{i}}\right)^{2}\left(\Gamma_{1}\right)^{-1}
\end{aligned}
$$


Поэтому,

$$
\begin{aligned}
\operatorname{tr}\left(\sum_{i=1}^{n} \frac{B_{i}^{1}(a)}{z-a_{i}}\right)^{2}= & \operatorname{tr}\left(\left(\Gamma_{1}^{\prime}\right)^{-1} \frac{\partial \Gamma_{1}^{\prime}}{\partial z} \sum_{i=1}^{n} \frac{{ }^{*} B_{i}(a)}{z-a_{i}}+\sum_{i=1}^{n} \frac{{ }^{*} B_{i}(a)}{z-a_{i}}\left(\Gamma_{1}^{\prime}\right)^{-1} \frac{\partial \Gamma_{1}^{\prime}}{\partial z}\right) \\
& +\operatorname{tr}\left(\sum_{i=1}^{n} \frac{{ }^{*} B_{i}(a)}{z-a_{i}}\right)^{2} .
\end{aligned}
$$

Так как все элементы матрицы $\left(\Gamma_{1}^{\prime}\right)^{-1} \partial \Gamma_{1}^{\prime} / \partial z$ равны нулю за исключением элемента с индексами $m, l$, который равен $-1 /\left(c b_{1}(a)\right)$, то мы получаем, что

$$
\operatorname{tr}\left(\sum_{i=1}^{n} \frac{B_{i}^{1}(a)}{z-a_{i}}\right)^{2}=\frac{-2}{c b_{1}(a)} \sum_{i=1}^{n} \frac{* b_{i}^{l m}(a)}{z-a_{i}}+\operatorname{tr}\left(\sum_{i=1}^{n} \frac{{ }^{*} B_{i}(a)}{z-a_{i}}\right)^{2} .
$$

Рассматривая вычеты обеих частей последнего соотношения в точке $a_{i}$, получаем

$$
\sum_{j \neq i} \frac{\operatorname{tr}\left(B_{i}^{1}(a) B_{j}^{1}(a)\right)}{a_{i}-a_{j}}=\frac{-1}{c b_{1}(a)}{ }^{*} b_{i}^{l m}(a)+\sum_{j \neq i} \frac{\operatorname{tr}\left({ }^{*} B_{i}(a)^{*} B_{j}(a)\right)}{a_{i}-a_{j}} .
$$

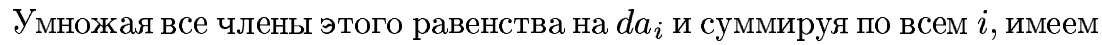

$$
\begin{aligned}
& \frac{1}{2} \sum_{i, j} \frac{\operatorname{tr}\left(B_{i}^{1}(a) B_{j}^{1}(a)\right) d\left(a_{i}-a_{j}\right)}{a_{i}-a_{j}}=\frac{-1}{c b_{1}(a)} \sum_{i=1}^{n}{ }^{*} b_{i}^{l m}(a) d a_{i} \\
& \quad+\frac{1}{2} \sum_{i, j i \neq j} \frac{\operatorname{tr}\left({ }^{*} B_{i}(a)^{*} B_{j}(a)\right) d\left(a_{i}-a_{j}\right)}{a_{i}-a_{j}} .
\end{aligned}
$$

Согласно (9) и (11) получаем, что

$$
\frac{-1}{c b_{1}(a)} \sum_{i=1}^{n}{ }^{*} b_{i}^{l m}(a) d a_{i}=d \log \left(b_{1}(a)\right)
$$

и окончательно,

$$
\begin{aligned}
& \frac{1}{2} \sum_{i, j} \frac{\operatorname{tr}\left(B_{i}^{1}(a) B_{j}^{1}(a)\right) d\left(a_{i}-a_{j}\right)}{a_{i}-a_{j}} \\
& \quad=d \log \left(b_{1}(a)\right)+\frac{1}{2} \sum_{i, j} \frac{\operatorname{tr}\left({ }^{*} B_{i}(a)^{*} B_{j}(a)\right) d\left(a_{i}-a_{j}\right)}{a_{i}-a_{j}} .
\end{aligned}
$$

Так как по построению форма

$$
\theta^{*}=\left(\frac{1}{2} \sum_{i, j} \frac{\operatorname{tr}\left({ }^{*} B_{i}(a)^{*} B_{j}(a)\right) d\left(a_{i}-a_{j}\right)}{a_{i}-a_{j}}\right)
$$

голоморфна и замкнута в некоторой окрестности $U^{*}$ точки $a^{*}$, то найдется голоморфная не зануляющаяся в этой окрестности функция $f^{*}(a)$ такая, что $d \log \left(f^{*}\right)=\theta^{*}$. Следовательно,

$$
\theta^{1}=\frac{1}{2} \sum_{i, j} \frac{\operatorname{tr}\left(B_{i}^{1}(a) B_{j}^{1}(a)\right) d\left(a_{i}-a_{j}\right)}{a_{i}-a_{j}}=d \log \left(f^{*}(a) b_{1}(a)\right) .
$$


Применив нужное число раз описанную процедуру, мы придем к исходной системе (3) и получим в $U^{*}$

$$
\theta=\frac{1}{2} \sum_{i, j} \frac{\operatorname{tr}\left(B_{i}(a) B_{j}(a)\right) d\left(a_{i}-a_{j}\right)}{a_{i}-a_{j}}=d \log \left(f^{*}(a) \tau^{*}(a)\right)
$$

для функции $\tau^{*}$ из (12).

Рассмотрим покрытие $\left\{U_{j}\right\}$ пространства $Z$ такое, что для каждого $U_{j}$ найдется голоморфная функция $\tau_{j}(a)$ с условием

$$
\theta=d \log \left(\tau_{j}(a)\right)
$$

и если $U_{j} \cap \Theta=\varnothing$, то соответствующая функция не зануляется.

В пересечении $U_{j} \cap U_{i}$ имеем $\tau_{j}=c_{j i} \tau_{i}$ с постоянными ненулевыми $c_{j i}$. Так как пространство $Z$ односвязно, то найдутся постоянные функции $c_{i}$ такие, что $c_{j}=c_{j i} c_{i}$ в $U_{j} \cap U_{i}$, и мы приходим к глобальной функции $\tau$, равной $\tau_{j} c_{j}$ в $U_{j}$ и такой, что

$$
\theta=d \log (\tau(a))
$$

в $Z$. Таким образом, мы передоказали следующую известную теорему Мивы из [4].

Теорема 1. Множсество $\Theta$ подвижных особых точек уравнения Шлезингера (1), (2) является множеством нулей функиии $\tau$, голоморфной на универсальном накрытии $Z$ пространства $\mathbb{C}^{n} \backslash \bigcup_{i=1}^{n}\left\{a_{i}-a_{j}=0\right\}$ и такой, что

$$
\frac{1}{2} \sum_{i, j} \frac{\operatorname{tr}\left(B_{i}(a) B_{j}(a)\right) d\left(a_{i}-a_{j}\right)}{a_{i}-a_{j}}=d \log (\tau(a)) .
$$

\section{СПИСОК ЦИТИРОВАННОЙ ЛИТЕРАТУРЫ}

[1] Болибрух А.А. Об изомонодромных слияниях фуксовых особенностей // Тр. МИАН. 1998. T. 221. C. $127-142$.

[2] Malgrange B. Sur les déformations isomonodromiques, I. Singularités régulières // Progr. Math. 1983. V. 37. P. 401-426.

[3] Bolibruch A. A. On orders of movable poles of the Schlesinger equation // J. Dynam. Control Systems. 2000. V. 6. №1. P. 57-74.

[4] Jimbo M., Miwa T. Monodromy preserving deformations of linear ordinary differential equations II // Phys. D. 1981. V. 2. P. 407-448.

[5] Оконек К., Шнейдер М., Шпиндлер Х. Векторные расслоения на комплексном проективном пространстве. М.: Мир, 1984.

[6] Bolibruch A. A. Vector bundles associated with monodromies and asymptotics of Fuchsian systems // J. Dynam. Control Systems. 1995. V. 1. №1. P. 229-252. 